\title{
An anatomical investigation of the superficial and deep palmar arches
}

\author{
S. Singh, L. Lazarus, B.Z. De Gama, K.S. Satyapal \\ Department of Clinical Anatomy, School of Laboratory Medicine and Medical Sciences, College of Health Sciences, \\ Westville Campus, University of KwaZulu Natal, Durban, South Africa
}

[Received: 16 April 2015; Accepted: 29 June 2015]

Background: The superficial palmar arch (SPA) and deep palmar arch (DPA) provide the dominant vascular supply to the hand. The SPA is considered to be highly variable and can be classified as either complete or incomplete. The simplest definition states that the anastomosis between the vessels contributing to the arch represent a complete arch, while an incomplete arch is described as characterised by an absence of anastomosis between the vessels contributing to it. This study aimed to describe the anatomical landmarks, formation and branching patterns of the SPA and DPA. In this study, the SPA and DPA were dissected in 50 specimens ( $n=100$ adult hands), respectively.

Materials and methods: A complete SPA was observed in 92\% of specimens and classified into three types. In Type A (44\%), the SPA was formed by the anastomosis of the superficial palmar branch of the radial artery with the ulnar artery. Type B (46\%) was formed by the ulnar artery alone and Type C (2\%) was formed by anastomosis of the ulnar artery with the superficial palmar branch of the radial artery and the persistent median artery.

Results: An incomplete SPA was observed in $8 \%$ of the specimens and divided into three types formed by the radial and ulnar arteries. The DPA was divided into five types viz. Type $G(72 \%)$, where the DPA was formed by anastomosis of the deep palmar branch of the radial artery (DPBRA) with the deep palmar branch of the ulnar artery (DPBUA). Type $H(12 \%)$, was formed by anastomosis of the DPBRA, the DBUA and the interosseous artery. Type I (8\%), was formed by the anastomosis of the DPBRA with the superior and inferior DPBUA. Type J (4\%), the deep ulnar artery had two branches whereby either one branch anastomosed with the DPBRA to form the DPA. Type K (4\%), the DBUA exhibited two deep branches with one branch anastomosing with the DPBRA to complete the DPA. Conclusions: The interosseous artery anastomosed with either the DPA or the additional DPBUA. Knowledge of the variability of the SPA and DPA is crucial for safe and successful hand surgeries. (Folia Morphol 2017; 76, 2: 219-225)

Key words: superficial palmar arch, deep palmar arch, radial artery, ulnar artery 


\section{INTRODUCTION}

Arterial dominance to the hand is provided by an intricate network of collateral flow referred to as the superficial (SPA) and deep (DPA) palmar arches [11]. According to Standring et al. [16], the ulnar artery is the main contributor of the SPA. One third of the SPA is formed by either the superficial palmar branch of the radial artery, the ulnar artery alone or by the arteria radialis indicis [16]. The DPA is formed primarily by the radial artery [16]. The terminal portion of the radial artery anastomoses with the deep palmar branch of the ulnar artery (DPBUA) to form the DPA [16]. The SPA curves laterally superficial to the long flexor tendons and deep to the palmar aponeurosis whereas the DPA is in contact with the bases of the metacarpals and curves medially deep to the long flexor tendons [13].

The SPA is highly variable with multifarious contributions from the ulnar, radial and persistent median arteries $[10,17]$. The variations of the SPA were first classified into two groups viz. complete and incomplete by Jachtschinski in 1897 (see [8]). The currently used classification and simplest definition states that the anastomosis between the vessels contributing to the arch represents a complete arch and an incomplete arch is described as having an absence of anastomosis between the vessels contributing to it [17]. In circumstances where the ulnar artery alone forms the SPA, the arch is also defined as complete provided that the ulnar artery reaches the space between the thumb and first digit [3].

Three or four arteries arise from the SPA and are referred to as common palmar digital arteries [5]. The common palmar digital arteries bifurcate into two proper palmar digital arteries (Fig. 1) [12]. Except for the radial side of the index finger and the ulnar side of the little finger, these arteries traverse the adjacent sides of all four medial fingers [12]. The thumb and radial side of the index finger are both supplied by branches of the radial artery called the arteria princeps pollicis and the arteria radialis indicis, respectively [12]. When an arterial trunk arising from the SPA provides origin to the arteria princeps pollicis and arteria radialis indicis, this trunk is considered as the first common palmar digital artery $[1,6]$.

The DPA travels transversely and more directly across the hand, whereas the SPA is semi-circular [11]. The DPA has less variability compared with the SPA [3]. The types of DPA are also classified as either complete or incomplete [15]. A complete DPA occurs when the terminal portion of the deep palmar branch of the

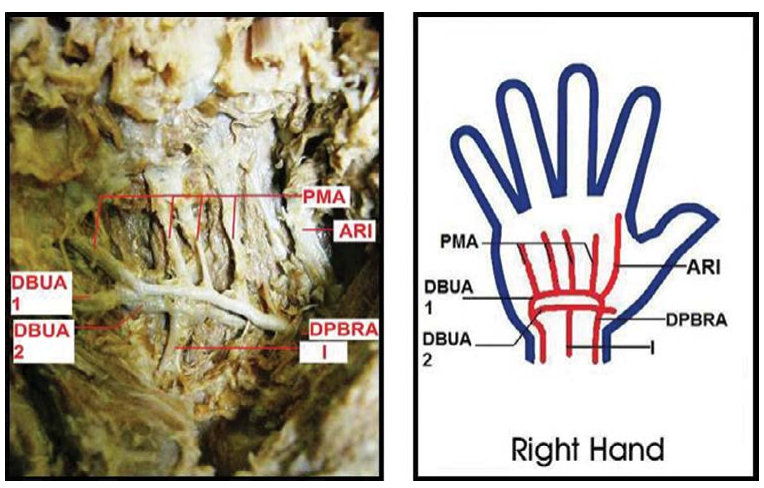

Figure 1. Right hand displaying cadaveric and schematic representation of Type $\mathrm{K}$ deep palmar arch (DPA) whereby the interosseous artery anastomosed with the second deep branch of ulnar artery; DPBRA - deep palmar branch of radial artery; DBUA 1 - first deep branch of ulnar artery contributing to the DPA; DBUA 2 second deep branch of ulnar artery; I - interosseous artery; $\mathrm{ARI}$ - arteria radialis indicis; PMA — palmar metacarpal arteries.

radial artery (DPBRA) anastomoses with the DPBUA [15]. An incomplete DPA occurs when the DPBRA has no connection with the DPBUA [15].

With advances in microsurgical techniques, knowledge of the frequency of vascular patterns, formation and branching of the palmar arches is essential for reconstructive hand surgery $[2,4]$.

This study thus aimed to describe the arteries forming the SPA and DPA, describe the branching pattern of the SPA and DPA, document the anatomical landmarks of the SPA and DPA and describe the anatomical variations of these arteries (if any).

\section{MATERIALS AND METHODS}

A study of 100 randomly selected adult hands was conducted at Clinical Anatomy, School of Laboratory Medicine and Medical Sciences at the University of KwaZulu-Natal Westville and the Nelson R Mandela Medical School Campuses in accordance with the National Health Act no 61 of 2003. The SPA was dissected in 50 specimens and the DPA was dissected in the remaining 50 specimens.

Using Grant's Dissector [18] as a guide, the palmar aspect of the hands was dissected and the course and branching patterns of the SPA and DPA was documented and images were recorded on a Canon Powershot A800 10 megapixel camera.

\section{RESULTS}

\section{Superficial palmar arch}

The SPA was divided into complete or incomplete types. The SPA was defined as complete when the ulnar 

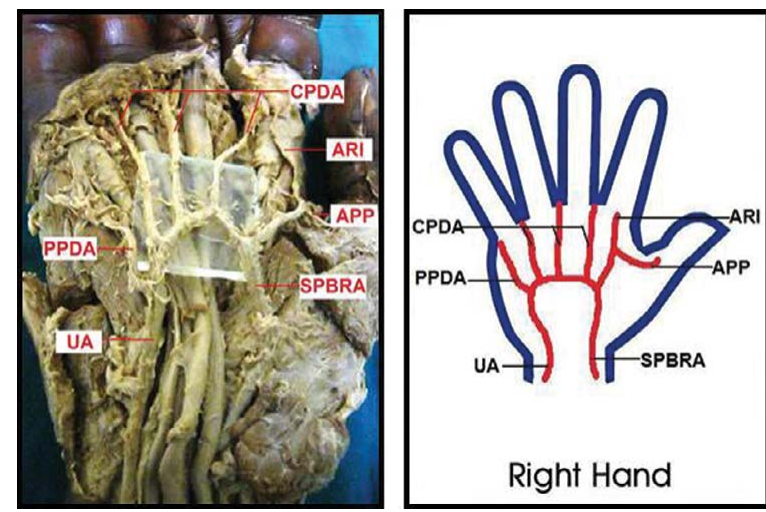

Right Hand

Figure 2. Cadaveric dissection and schematic representation showing Type A superficial palmar arch; SPBRA — superficial palmar branch of the radial artery; UA — ulnar artery; APP arteria princeps pollicis; ARI — arteria radialis indicis; CPDA common palmar digital arteries; PPDA — proper palmer digital artery to the little finger.
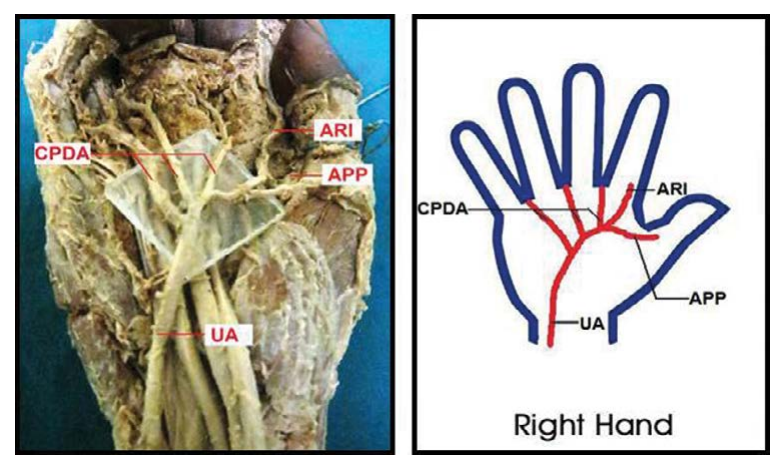

Figure 3. Cadaveric dissection and schematic representation showing Type B superficial palmar arch; UA — ulnar artery; APP — arteria princeps pollicis; ARI — arteria radialis indicis; CPDA — common palmar digital arteries.

artery anastomosed with either or both the superficial palmar branch of the radial and persistent median arteries or the ulnar artery reached the space between the thumb and the first digit. Where no such anastomosis occurs, the SPA was defined as incomplete.

\section{Complete types of SPA}

A complete SPA was encountered in $46 / 50$ (92\%) of specimens. The arches were divided into three types depending on the formation of the SPA, viz.

- Type A (Fig. 2) contained the superficial arches completed by the anastomosis of the superficial palmar branch of the radial artery with the ulnar artery. This type was observed in $22 / 50(44 \%)$ of specimens.

- Type B (Fig. 3) contained the complete superficial arches formed by the ulnar artery only. This type was observed in 23/50 (46\%) of specimens. In 19/23
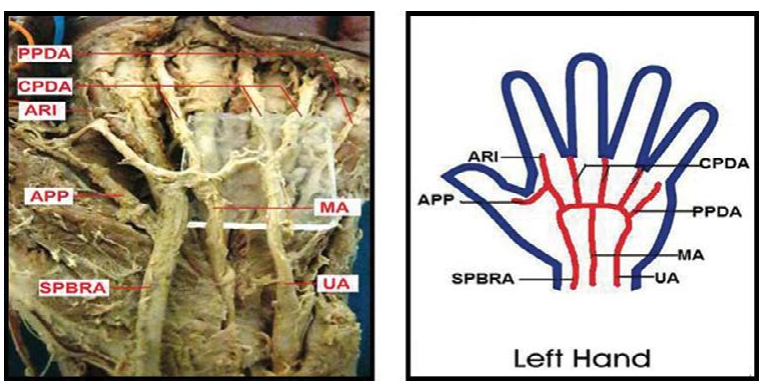

Figure 4. Cadaveric dissection and schematic representation showing Type $C$ superficial palmar arch; SPBRA - superficial palmar branch of the radial artery; UA - ulnar artery; MA persistent median artery; APP — arteria princeps pollicis; $\mathrm{ARI}$ - arteria radialis indicis; CPDA — common palmar digital arteries; PPDA — proper palmer digital artery to the little finger.

(83\%) of these specimens, the ulnar artery provided the branches supplying the thumb and index finger called the arteria princeps pollicis and arteria radialis indicis, respectively. The remaining $4 / 23(17 \%)$ provided the arteria princeps pollicis arteries; however, the arteria radialis indicis arteries were absent.

- Type C (Fig. 4) contained an SPA formed by the anastomosis of the superficial palmar branch of the radial artery, the ulnar artery and the persistent median artery. This type was observed in 1/50 (2\%) of specimens.

Within the Type A (radio-ulnar) superficial arches, $4 / 22(18 \%)$ variations were observed including two with unusual branching patterns. These variations were designated by the letter a, viz.

- Subtype al: in this variation, the third common palmar digital artery (between the ring and little finger) and the proper palmar digital artery to the little finger arose from a single trunk branching from the SPA. The first and second common palmar digital arteries arose normally from the SPA.

- Subtype all: in this variation, the common palmar digital arteries arose from a single trunk branching from the SPA. The proper palmar digital artery to the little finger arose normally from the SPA.

- Subtype alll: in this variation the SPA provided the arteria radialis indicis and did not give rise to the arteria princeps pollicis.

- Subtype alV: in this variation the SPA gave origin to the arteria princeps and the common palmar digital arteries arose normally from the SPA.

Within Type B (ulnar type), 2/23 (9\%) variations were observed in three specimens and designated by the letter $b$, viz. 
- Subtype bl: this type showed a variation whereby the first common palmar digital artery (between the index and middle finger) and the second common palmar digital artery (between the middle and ring finger) arose from a single trunk branching from the ulnar type SPA. The third common palmar digital artery and proper digital artery to the little finger arose normally from the SPA. The ulnar artery also provided the arteria princeps pollicis and arteria radialis indicis arteries.

- Subtype bll: in this variation the ulnar type SPA gave rise to the arteria princeps pollicis and not the arteria radialis indicis.

\section{Incomplete types of SPA}

An incomplete SPA was observed in $4 / 50(8 \%)$ of specimens. All incomplete arches were formed by the radial and ulnar arteries. Three types of incomplete superficial arches were observed, viz.

- Type D: the radial artery gave rise to the arteria princeps pollicis and arteria radialis indicis. The radial artery terminated by providing the first common palmar digital artery (between the index and middle fingers). The ulnar artery gave rise to the second common palmar digital artery (between the middle and ring fingers) and the proper palmar digital artery to the little finger. This type was observed in $1 / 4(25 \%)$ of specimens.

- Type E: the radial artery provided the arteria princeps pollicis and arteria radialis indicis. The radial artery also gave rise to the first common palmar digital artery (between the index and middle fingers). The second common palmar digital artery (between the middle and ring fingers), the third common palmar digital artery (between the ring and little fingers) and the proper palmar digital artery to the little finger arose from the ulnar artery. This type was observed in 2/4 (50\%) of specimens.

- Type F: the radial artery gave rise to the arteria princeps pollicis and arteria radialis indicis whereas the ulnar artery gave rise to all three common palmar digital arteries. The proper palmar digital artery to the little finger was not present. This type was observed in $1 / 4(25 \%)$ of specimens.

\section{Deep palmar arch}

The deep arches were classified as complete in all $50 / 50(100 \%)$ of specimens. The complete arches were defined as having an anastomosis between the arteries constructing the arch and an incomplete arch was defined by an absence of an anastomosis between the arteries constructing the arch. The deep arches were then divided into five types depending on the formation of the DPA, viz.

- Type G contained the deep arches completed by the anastomosis of the DPBRA with the deep branch of the ulnar artery. This type was observed in $36 / 50(72 \%)$ of specimens.

- Type $\mathrm{H}$ contained the deep arches formed by the DPBRA, the deep branch of the ulnar artery and additional supply from the interosseous artery. This type was observed in $6 / 50(12 \%)$ of specimens.

- Type I contained the deep arches whereby the deep branch of the ulnar artery divides into a superior and inferior branch which anastomoses with the DPBRA. This type was observed in $4 / 50$ (8\%) of specimens.

- Type J contained the deep arches whereby the deep branch of the ulnar artery exhibited two deep branches. One branch anastomosed with the DPBRA to form the DPA. The other branch followed the DPA to the thenar region. This type was observed in 2/50 (4\%) of specimens.

- Type K (Fig. 1) contained the deep arches whereby the deep branch of the ulnar artery exhibited two deep branches with one branch anastomosing with the DPBRA to complete the DPA. The interosseous artery anastomosed with either the DPA or the additional deep branch of the ulnar artery. This type was observed in $2 / 50(4 \%)$ of specimens.

\section{DISCUSSION}

\section{The superficial palmar arch}

In the present study the SPA was found to be less variable than the DPA. Jachtschinski in 1897 (see [8]) was the first to establish a system of classification for the SPA by separating the SPA into a complete or incomplete group [7]. This implied that a complete arch is represented by the anastomosis between the arteries constructing the arch such as the radial, ulnar and median arteries and when these arteries fail to anastomose the arch is referred to as incomplete [17].

In this study, the SPA was classified into complete and incomplete arches. The complete arches were encountered in $92 \%$ of specimens and included arches formed by the ulnar artery alone reaching the space between the thumb and the index finger agreeing with the findings of Loukas et al. [10] (90\%) and Bilge et al. [4] (86\%). In the ulnar predominant arches, the 
ulnar artery gave origin to the common palmar digital arteries as well as the arteria princeps pollicis and arteria radialis indicis which are typically branches of the radial artery that supply the thumb, therefore providing adequate arterial supply to the palm in the absence of the superficial palmar branch of the radial artery. This classification does not correlate with Al-Turk and Metcalf [1], Lippert and Pabst [9], Ruengsakulrach et al. [15] and Fazan et al. [6] all of whom classified the SPA as incomplete when formed by the ulnar artery alone.

Table 1 outlines the various types of SPA, its formation and incidences with the references and incidence in comparison to previous literature. The complete SPA observed in this study was divided into three types. The standard classification describing the SPA as completed by the anastomosis of the superficial palmar branch of the radial artery with the ulnar artery has been reported to be evident in as many as $62 \%$ of specimens in a study by Bilge et al. [4] or as few as $10 \%$ of specimens according to the study by Ruengsakulrach et al. [15]. The present study observed the standard classification in $44 \%$ of specimens and was classified as Type A correlating with Loukas et al. [10] $40 \%$ in their type S-I classification of the SPA.

Within Type $A, 18 \%$ of specimens exhibited unusual variations that involved different branching patterns of the common and proper palmar digital arteries. These variations did not concur with other authors and were divided into four subtypes. In the first subtype, Subtype al, the third common palmar digital artery and the proper palmar digital artery to the little finger arose from a single trunk branching from the SPA. The first and second common palmar digital arteries arose normally from the SPA. In Subtype all, three common palmar digital arteries arose from a single trunk branching from the SPA and the proper palmar digital artery to the little finger arose normally from the SPA. The SPA in Subtype alll gave origin to the arteria radialis indicis and the common palmar digital arteries arose normally from the SPA. In Subtype alV, the arteria princeps pollicis originated from the SPA and the common palmar digital arteries arose normally from the SPA.

Type B was the most common variant of SPA in this study. This type contained the SPA formed by the ulnar artery alone and was observed in $46 \%$ of specimens differing widely from Loukas et al. [10] 35\% in their S-II classification of this formation. Within Type B, $9 \%$ of specimens exhibited unusual varia-
Table 1. Outline of the superficial palmar arch (SPA) types compared with the literature

\begin{tabular}{|c|c|}
\hline Author (year) & Incidence (\%) \\
\hline \multicolumn{2}{|c|}{$\begin{array}{l}\text { Complete type: A } \\
\text { Superficial palmar branch of the radial artery and the ulnar artery }\end{array}$} \\
\hline Al-Turk and Metcalf (1984) & 78 \\
\hline Lippert and Pabst (1985) & 35 \\
\hline Ruengsakulrach et al. (2001) & 10 \\
\hline Loukas et al. (2005) & 40 \\
\hline Bilge et al. (2006) & 62 \\
\hline Feigl et al. (2011) & 16.1 \\
\hline This study & 44 \\
\hline \multicolumn{2}{|c|}{$\begin{array}{c}\text { Complete type: B } \\
\text { Exclusively the ulnar artery only }\end{array}$} \\
\hline Loukas et al. (2005) & 35 \\
\hline Bilge et al. (2006) & 14 \\
\hline This study & 46 \\
\hline \multicolumn{2}{|c|}{$\begin{array}{c}\text { Complete type: C } \\
\text { Radial, ulnar and median arteries }\end{array}$} \\
\hline Al-Turk and Metcalf (1984) & 2 \\
\hline Lippert and Pabst (1985) & 1 \\
\hline Loukas et al. (2005) & 6.1 \\
\hline Feigl et al. (2011) & 0.4 \\
\hline This study & 2 \\
\hline \multicolumn{2}{|c|}{$\begin{array}{l}\text { Incomplete type: D } \\
\text { Radial and ulnar artery }\end{array}$} \\
\hline Lippert and Pabst (1985) & 3 \\
\hline This study & 2 \\
\hline \multicolumn{2}{|c|}{$\begin{array}{l}\text { Incomplete type: } \mathbf{E} \\
\text { Radial and ulnar arteries }\end{array}$} \\
\hline Al-Turk and Metcalf (1984) & 2 \\
\hline Bilge et al. (2006) & 6 \\
\hline This study & 4 \\
\hline \multicolumn{2}{|c|}{$\begin{array}{l}\text { Incomplete type: } \mathbf{F} \\
\text { Radial and ulnar arteries }\end{array}$} \\
\hline Al-Turk and Metcalf (1984) & 8 \\
\hline This study & 2 \\
\hline
\end{tabular}

tions. These variations did not correspond with other authors and were divided into two subtypes. Within Subtype bl, the first common palmar digital artery and the second common palmar digital artery arose from a single trunk branching from the SPA and the ulnar artery also provided the arteria princeps pollicis and arteria radialis indicis. In Subtype bll, the ulnar artery gave origin to the arteria princeps pollicis. The common palmar digital arteries and proper palmar digital artery to the little finger arose normally from 
the SPA. The third type of complete arch presented in this study, Type C, contained an arch completed by the superficial palmar branch of the radial artery, the ulnar artery and the persistent median artery. This type was observed in $2 \%$ of specimens differing slightly from Loukas et al. [10] in their S-IV classification.

The incomplete arches observed in this study were defined as having no anastomosis with the vessels constructing the arch. The incomplete arch was observed in $8 \%$ of specimens and classified into three types: Type D, Type E, Type F, depending on the branching pattern of the common palmar digital arteries and the proper palmar digital artery to the little finger. These results agreed with Loukas et al. [10] (10\%) and Bilge et al. [4] (14\%).

\section{The deep palmar arch}

The present study found the DPA to be $100 \%$ complete in all specimens, concurring with the results from a study by Loukas et al. [10] (100\%). In this study a complete DPA was defined as an anastomosis between the radial and ulnar arteries and when the anastomosis was not present the arch was defined as incomplete. The DPA was divided into five types in the present study. Table 2 outlined the various types of DPA, its formation and incidence in comparison to previous literature.

In Type $G$ of the DPA, the DPBRA anastomosed with DPBUA and was observed in $72 \%$ of specimens. This agreed with results obtained by Lippert and Pabst [9] who observed $79 \%$ and Loukas et al. [10] who reported $60 \%$ of this type of DPA.

The Type H DPA consisted of arches formed by the DPBRA, DPBUA and the interosseous artery. This type was observed in $12 \%$ of specimens and only agreed with Lippert and Pabst [9] who described 5\% of this type.

In Type I of the DPA, DPBUA divided into a superior and inferior branch which both anastomosed with the DPBRA. These results were observed in $8 \%$ of specimens and concurred with Olave and Prates [14] (13\%).

The Type J DPA described an anastomosis between DPBRA and the first of two deep branches of the ulnar artery and was observed in $4 \%$ of specimens. This type agreed with Lippert and Pabst [9] (13\%) and disagreed with Olave and Prates [14] (46.7\%).

In Type K DPA, the interosseous artery anastomosed with one of two deep branches of the ulnar artery. This type was observed in $4 \%$ of specimens and has not been reported by other authors.
Table 2. Outline of the deep palmar arch (DPA) types compared with the literature

\begin{tabular}{|c|c|}
\hline Author (year) & Incidence (\%) \\
\hline \multicolumn{2}{|c|}{$\begin{array}{c}\text { Type: } \mathbf{G} \\
\text { Deep palmar branch of radial and ulnar artery }\end{array}$} \\
\hline Lippert and Pabst (1985) & 79 \\
\hline Loukas et al. (2005) & 60 \\
\hline This study & 72 \\
\hline \multicolumn{2}{|c|}{$\begin{array}{c}\text { Type: } \mathbf{H} \\
\begin{array}{c}\text { Deep palmar branch of radial artery, deep branch of ulnar artery } \\
\text { and interosseous artery }\end{array}\end{array}$} \\
\hline Lippert and Pabst (1985) & 5 \\
\hline This study & 12 \\
\hline \multicolumn{2}{|c|}{$\begin{array}{c}\text { Type: I } \\
\begin{array}{c}\text { Deep palmar branch of radial artery, superior and inferior } \\
\text { deep branch of ulnar artery }\end{array}\end{array}$} \\
\hline Olave and Prates (1998) & 13 \\
\hline This study & 8 \\
\hline \multicolumn{2}{|c|}{$\begin{array}{c}\text { Type: } \mathrm{J} \\
\text { Deep palmar branch of radial artery and the first of two } \\
\text { deep branches of the ulnar artery }\end{array}$} \\
\hline Lippert and Pabst (1985) & 13 \\
\hline This study & 4 \\
\hline \multicolumn{2}{|c|}{$\begin{array}{c}\text { Type: } K \\
\begin{array}{c}\text { Deep palmar branch of the radial artery, one of two deep branches } \\
\text { of the ulnar artery and the interosseous artery }\end{array}\end{array}$} \\
\hline This study & 4 \\
\hline
\end{tabular}

No incomplete deep arches were present in this study disagreeing with Lippert and Pabst [9] who described an incidence of $3 \%$ of incomplete arches.

The contribution of the radial and ulnar arteries to the superficial and deep palmar arches is immensely clinically significant. In particular the radial artery contribution to the thenar muscles and fingers is crucial when considering harvesting of the radial artery [10]. Successful harvesting of the radial artery necessitates a complete palmar arch and patency of the ulnar artery [3]. Hence pre-operative screening is imperative to avoid ischaemia [10]. Although radial and ulnar dominance was not determined by this study, the greater part of the SPA dissections and all the DPA dissections exhibited a collateral circulation between the radial and ulnar arteries. Anatomically this indicates the removal of one of these arteries will be safe. Ruengsakulrach et al. [15] suggested the use of the modified Allen test or Doppler ultrasonography to determine the competence of the palmar collateral circulation. 


\section{CONCLUSIONS}

This study aimed to acquire a greater understanding of the variability of the formation and branching patterns of the SPA and DPA. Both the complete and incomplete types of SPA ascertained were classified into the radial-ulnar type, ulnar predominant type and radial-ulnar-median type. The DPA exhibited a greater variability than the SPA resulting in its division into five differing complete types. The DPA types included the deep radial-ulnar type, the deep radialulnar-interosseous type, the deep radial-superior and inferior ulnar type, the deep radial and the first of two deep branches of the ulnar type and the deep radial and one of two deep branches of the ulnar, interosseous type. The comprehension of the variations of the arteries forming the palmar arches, particularly the radial artery, contributes to the ever expanding knowledge base facilitating surgical approaches.

\section{REFERENCES}

1. Al-Turk M, Metcalf WK. A study of the superficial palmar arteries using the Doppler Ultrasonic Flowmeter. J Anat. 1984; 138 (Pt 1): 27-32, indexed in Pubmed: 6706837.

2. Aughsteen AA. Case report of a new variant of double incomplete superficial palmar arch. Anat Sci Int. 2012; 87(1): 56-59, doi: 10.1007/s12565-011-0107-9, indexed in Pubmed: 21559884.

3. Baetz L, Satiani B. Palmar arch identification during evaluation for radial artery harvest. Vasc Endovascular Surg. 2011; 45(3): 255-257, doi: 10.1177/1538574411399159, indexed in Pubmed: 21478246.

4. Bilge O, Pinar Y, Ozer MA, et al. A morphometric study on the superficial palmar arch of the hand. Surg Radiol Anat. 2006; 28(4): 343-350, doi: 10.1007/s00276-006-0109-9, indexed in Pubmed: 16642281.

5. Brzezinski M, Luisetti T, London MJ. Radial artery cannulation: a comprehensive review of recent anatomic and physiologic investigations. Anesth Analg. 2009; 109(6): 1763-1781, doi: 10.1213/ANE.0b013e3181bbd416, indexed in Pubmed: 19923502.
6. Fazan VP, Borges CT, Da Silva JH, et al. Superficial palmar arch: an arterial diameter study. J Anat. 2004; 204(4): 307-311, doi: 10.1111/j.0021-8782.2004.00281.x, indexed in Pubmed: 15061757.

7. Feigl GC, Petrac M, Pixner T, et al. The superficial palmar arch and median artery as an example of misleading results due to a small number of investigated specimens or the use of different classifications. Ann Anat. 2012; 194(4): 389-395, doi: 10.1016/j.aanat.2011.10.013, indexed in Pubmed:22196998.

8. Jaschtschinski SN. Morphologie und Topographie des Arcus volaris sublimes und prefundus des Menchen. Anat Heff. 1897; 7: 161-188.

9. Lippert $H$, Pabst R. Arterial variations in man: classification and frequency. J.F. Bergmann (Springer) 1985: 74-75.

10. Loukas M, Holdman D, Holdman S. Anatomical variations of the superficial and deep palmar arches. Folia Morphol (Warsz). 2005; 64(2): 78-83, indexed in Pubmed: 16121323.

11. McLean KM, Sacks JM, Kuo YR, et al. Anatomical landmarks to the superficial and deep palmar arches. Plast Reconstr Surg. 2008; 121(1): 181-185, doi: 10.1097/01. prs.0000293863.45614.f9, indexed in Pubmed: 18176219.

12. Mookambica RV, Nair V, Nair R, et al. Incomplete superficial palmar arch. Int J Anat Variations. 2012.; 3: 65-66.

13. Moore KL, Dalley AF, Agur AMR. Clinically Oriented Anatomy. Sixth Edition. Lippincott Williams and Wilkins (Wolters Kluwer) 2010: 775-782.

14. Olave E, Prates JC. Deep palmar arch patterns in Brazilian individuals. Surg Radiol Anat. 1999; 21(4): 267-271, indexed in Pubmed: 10549084.

15. Ruengsakulrach $P$, Eizenberg $N$, Fahrer $C$, et al. Surgical implications of variations in hand collateral circulation: anatomy revisited. J Thorac Cardiovasc Surg. 2001; 122(4): 682-686, doi: 10.1067/mtc.2001.116951, indexed in Pubmed: 11581598.

16. Standring S, Borley NR, Collins P. et al. Gray's Anatomy, The Anatomical Basis of Clinical Practice. Fortieth edition. Churchill Livingstone (Elsevier) 2009: 2321-2330.

17. Takkallapalli A, Kalbande S, Dombe D, et al. 2011. Variations in the formation of superficial palmar arch and its clinical significance in hand surgeries. Int J Biol Med Res. 2011; 2(2): 543-546.

18. Tank PW. Grant's Dissector. 14th Ed. Lippincott Williams and Wilkins (Wolters Kluwer) 2009: 41-46. 